\title{
The Better Half of Selling Separately
}

\author{
SERGIU HART, The Hebrew University of Jerusalem \\ PHILIP J. RENY*, University of Chicago
}

\begin{abstract}
Separate selling of two independent goods is shown to yield at least $62 \%$ of the optimal revenue, and at least $73 \%$ when the goods satisfy the Myerson regularity condition. This improves the $50 \%$ result of Hart and Nisan [2017, originally circulated in 2012].
\end{abstract}

CCS Concepts: - Theory of computation $\rightarrow$ Theory and algorithms for application domains; Algorithmic game theory and mechanism design; Computational pricing and auctions;

Additional Key Words and Phrases: Selling two independent goods, monopolistic pricing, optimal revenue, selling separately, approximation bound

\section{ACM Reference format:}

Sergiu Hart and Philip J. Reny. 2019. The Better Half of Selling Separately. ACM Trans. Econ. Comput. 7, 4, Article 18 (December 2019), 18 pages.

https://doi.org/10.1145/3369927

\section{INTRODUCTION}

One of the most celebrated aspects of Myerson's [1981] optimal auction result is that it provides an economic explanation for the ubiquitous use of the four standard auction forms. Strictly speaking, however, Myerson's results apply only to cases in which a seller is selling a single good. Because many sellers sell multiple goods, extending Myerson's analysis to the multi-good case has long been considered a critical next step. But the multi-good monopoly problem has resisted a complete solution for over 35 years and by now it is well understood to be an extremely difficult problem. Worse still, it is known that the optimal solution must typically be quite complex and very often requires buyers to purchase randomized contracts. ${ }^{1}$ And therein lies the difficulty, because we do not often, if ever, observe complex or randomized selling mechanisms in practice. This raises the obvious question, Why not?

\footnotetext{
${ }^{1}$ Examples where randomization increases the revenue appear in the literature, starting with Thanassoulis [2004]. See Hart and Reny [2015] for the analysis of a particularly simple such example, and references to this literature.

Previous versions: October 2018, December 2017 (https://arxiv.org/abs/1712.08973), November 2016. We thank Noam Nisan for useful discussions, and the editors and referees for their helpful suggestions and comments.

${ }^{*}$ Research partially supported by National Science Foundation grant SES-1724747.

Authors' addresses: S. Hart, Federmann Center for the Study of Rationality, The Hebrew University of Jerusalem, Feldman Building, Givat Ram Campus, 9190401 Israel; email: hart@huji.ac.il; P. J. Reny, Kenneth C. Griffin Department of Economics, University of Chicago, 1126 East 59th Street, Chicago, IL 60637, U.S.A; email: preny@uchicago.edu.

Permission to make digital or hard copies of all or part of this work for personal or classroom use is granted without fee provided that copies are not made or distributed for profit or commercial advantage and that copies bear this notice and the full citation on the first page. Copyrights for components of this work owned by others than the author(s) must be honored. Abstracting with credit is permitted. To copy otherwise, or republish, to post on servers or to redistribute to lists, requires prior specific permission and/or a fee. Request permissions from permissions@acm.org.

(C) 2019 Copyright held by the owner/author(s). Publication rights licensed to ACM.

2167-8375/2019/12-ART18 \$15.00

https://doi.org/10.1145/3369927
} 
One reason that we may not observe the kinds of complex mechanisms that full optimality dictates is that relatively simple mechanisms may suffice for generating much of the revenue that could ever be generated. Thus, a complementary approach to the research program initiated in Myerson [1981] is to search for relatively simple mechanisms that yield a significant fraction of the revenue that is generated by a fully optimal mechanism. ${ }^{2}$ The present article represents a modest contribution to this program.

We consider a single seller who has one unit each of two indivisible goods. The two goods need not be identical. The seller, whose value for the two goods is zero, can offer to sell the goods to a single buyer. The buyer's two values, one value for each of the two goods, are unknown to the seller but are known to be independently distributed (so, we say that the two goods are independent). The buyer is risk neutral and has preferences that are additive in the values and (negatively so) in the price paid. Even in this most basic case, there is no known characterization of the optimal selling mechanism, though it is known that the optimal mechanism can display unusual properties. ${ }^{3} \mathrm{We}$ ask, What fraction of the optimal revenue can the seller guarantee by selling each of the two goods separately, i.e., by posting a Myerson-optimal price for each of the goods?

In the context of a general analysis with any finite number of independent goods, Hart and Nisan [2017] ${ }^{4}$ show in particular that, by selling two independent goods separately, the seller can guarantee at least $50 \%$ of the optimal revenue but cannot guarantee more than ${ }^{5} 78 \%$. A nice feature of the $50 \%$ revenue guarantee is that its proof is relatively simple. In part, this simplicity arises from the rather generous bounds that are established at various steps. While it seems clear that the bounds employed in the Hart-Nisan proof are "much" too generous, tightening them as we do here requires a surprising amount of additional effort. Hart and Nisan also show that if, in addition, the buyer's two independent values are identically distributed, then the revenue guarantee is at least $73 \%$, which is tantalizingly close to the $78 \%$ upper limit.

Our main result significantly improves upon the Hart-Nisan $50 \%$ guarantee, and shows that their $73 \%$ guarantee with identically distributed values can also be obtained when the buyer's value distributions satisfy Myerson-regularity. None of our results require the two values to be identically distributed.

Main Result. For any two independent goods, selling each good separately at its optimal one-good price guarantees at least $\sqrt{e} /(\sqrt{e}+1) \approx 62 \%$ of the optimal revenue. Furthermore, if the buyer's two value distributions each satisfy Myerson-regularity, then the guaranteed fraction of optimal revenue increases to $e /(e+1) \approx 73 \%$.

This is stated below as Theorems 7 and 9 .

To summarize the known bounds on the guaranteed fraction of optimal revenue (GFOR) from selling separately two goods: when the goods are independent, the GFOR is at least $62 \%$; when they are independent and either Myerson-regular or identically distributed, the GFOR is at least $73 \%$; in all these three cases, the GFOR is at most $78 \%$; and, when the goods are not necessarily independent, the GFOR drops all the way down to zero [Hart and Nisan 2019]. ${ }^{6}$

\footnotetext{
${ }^{2}$ See Chawla et al. [2007] (in the related unit-demand setup); Hart and Nisan [2012/2017] provides an overview of this literature.

${ }^{3}$ Such as being non-monotonic: increasing the buyer's valuations may well decrease the seller's optimal revenue; see Hart and Reny [2015].

${ }^{4}$ Originally circulated in 2012 (EC-2012).

${ }^{5}$ Hart and Nisan [2017] establish the 78\% upper bound with an explicit example in which it is optimal to sell two independent and identically distributed goods as a bundle (these goods satisfy the Myerson-regularity condition).

${ }^{6}$ For more than two goods a similar result is due to Briest et al. [2015].
} 


\subsection{Some Related Work}

There is by now a vast literature in game theory, economics, and computer science that deals with the (optimal) selling of multiple goods. While that literature is too large to survey here, the reader may wish to consult the literature section in, say, Hart and Nisan [2017] for an overview of prior work; see also the references in the Introduction section above. We will mention here the result of Babaioff et al. [2014] the better option between selling the goods separately and selling them as the bundle of all goods yields a GFOR that is bounded away from zero (specifically, $\geq 1 / 6$ ) for any number of independent goods. Recently, in the case of two independent goods, Babaioff et al. [2018] have shown that separate selling yields at least $78 \%$ of the optimal deterministic revenue, and that this bound is tight. In the related setup of a unit-demand buyer (who desires to buy only one good, rather than having an additive value over bundles of goods), Chawla et al. [2010, Theorem 5] show a GFOR of $1 / 4$ for the separate selling of any number of independent goods. Finally, Daskalakis et al. [2017] provide a duality-based characterization of the revenue-optimizing mechanism for multiple goods.

\subsection{Organization of the Article}

The article is organized as follows: Section 2 presents the model, defines the appropriate concepts, and provides some preliminary results. Section 3 gives an outline of the proof. The proof itself consists of a general decomposition result (Proposition 4 in Section 4) and an estimate of the crucial term there (Proposition 6 in Section 5), which, when combined, give the first part of the Main Theorem, namely, the general 62\% bound (Theorem 7 in Section 6). Section 7 proves the second part of the Main Theorem, namely, the 73\% bound for regular distributions (Theorem 9), together with some additional results. Appendix A provides a general result on the continuity of the revenue with respect to valuations (which is of independent interest), and Appendix B gives a simple illustration of the use of nonsymmetric diagonals.

\section{PRELIMINARIES}

\subsection{The Model}

The basic model is standard, and the notation follows Hart and Reny [2015] and Hart and Nisan [2017], which the reader may consult for further details and references.

One seller (or "monopolist") is selling a number $k \geq 1$ of goods (or "items," "objects," and so on) to one buyer.

The goods have no value or cost to the seller. Let $x_{1}, x_{2}, \ldots, x_{k} \geq 0$ be the buyer's values for the goods. The value for getting a set of goods is additive: getting the subset $I \subseteq\{1,2, \ldots, k\}$ of goods is worth $\sum_{i \in I} x_{i}$ to the buyer (and so, in particular, the buyer's demand is not restricted to one good only). The valuation of the goods is given by a random variable $X=\left(X_{1}, X_{2}, \ldots, X_{k}\right)$ that takes values in $\mathbb{R}_{+}^{k}$ (we thus assume that valuations are always nonnegative); we will refer to $X$ as a $k$-good random valuation. The realization $x=\left(x_{1}, x_{2}, \ldots, x_{k}\right) \in \mathbb{R}_{+}^{k}$ of $X$ is known to the buyer, but not to the seller, who knows only the probability distribution of $X$ (which may be viewed as the seller's belief); we refer to a buyer with valuation $x$ also as a buyer of type $x$. The buyer and the seller are assumed to be risk neutral and to have quasi-linear utilities.

The objective is to maximize the seller's (expected) revenue.

By the Revelation Principle [Myerson 1981], it is without loss of generality to restrict attention to "direct mechanisms" that are "incentive compatible." A directmechanism $\mu$ consists of a pair of functions $^{7}(q, s)$, where $q=\left(q_{1}, q_{2}, \ldots, q_{k}\right): \mathbb{R}_{+}^{k} \rightarrow[0,1]^{k}$ and $^{8} s: \mathbb{R}_{+}^{k} \rightarrow \mathbb{R}$. If the buyer reports a

\footnotetext{
${ }^{7}$ All functions in this article are assumed to be Borel measurable [cf. Hart and Reny 2015, footnotes 10 and 48].

${ }^{8}$ Without loss of generality, any mechanism can always be extended to the whole space $\mathbb{R}_{+}^{k}$; see Hart and Reny [2015].
} 
valuation vector $x \in \mathbb{R}_{+}^{k}$, then $q_{i}(x) \in[0,1]$ is the probability that the buyer receives good $i$ (for $i=1,2, \ldots, k)$, and $s(x)$ is the payment that the seller receives from the buyer. When the buyer reports his value $x$ truthfully, his payoff is ${ }^{10} b(x)=\sum_{i=1}^{k} q_{i}(x) x_{i}-s(x)=q(x) \cdot x-s(x)$, and the seller's payoff is $s(x)$.

The mechanism $\mu=(q, s)$ satisfies individual rationality (IR) if $b(x) \geq 0$ for every ${ }^{11} x \in \mathbb{R}_{+}^{k}$; it satisfies incentive compatibility (IC) if $b(x) \geq q(\tilde{x}) \cdot x-s(\tilde{x})$ for every alternative report $\tilde{x} \in \mathbb{R}_{+}^{k}$ of the buyer when his value is $x$, for every $x \in \mathbb{R}_{+}^{k}$; and it satisfies no positive transfer (NPT) if $s(x) \geq 0$ for every $x \in \mathbb{R}_{+}^{k}$ (which, together with IR, implies that $s(0)=b(0)=0$ ).

The (expected) revenue of a mechanism $\mu=(q, s)$ from a buyer with random valuation $X$, which we denote by $R(\mu ; X)$, is the expectation of the payment received by the seller; i.e., $R(\mu ; X)=$ $\mathbb{E}[s(X)]$. We now define:

- $\operatorname{Rev}(X)$, the optimal revenue, is the maximal revenue that can be obtained: $\operatorname{Rev}(X):=$ $\sup _{\mu} R(\mu ; X)$, where the supremum is taken over all mechanisms $\mu$ that satisfy IR and IC.

When there is only one good, i.e., when $k=1$, Myerson's [1981] result is that

$$
\operatorname{Rev}(X)=\sup _{p \geq 0} p \cdot(1-F(p)),
$$

where $F$ is the cumulative distribution function of $X$. Optimal mechanisms correspond to the seller "posting" a price $p$ and the buyer buying the good for the price $p$ whenever his value is at least $p$; in other words, the seller makes the buyer a "take-it-or-leave-it" offer to buy the good at price $p$.

Besides the maximal revenue $\operatorname{Rev}(X)$, we consider what can be obtained from the simple class of mechanisms that sell each good separately.

- SRev $(X)$, the separate revenue, is the maximal revenue that can be obtained by selling each good separately. Thus,

$$
\operatorname{SREv}(X)=\operatorname{Rev}\left(X_{1}\right)+\operatorname{Rev}\left(X_{2}\right)+\ldots+\operatorname{Rev}\left(X_{k}\right) .
$$

The separate revenue is obtained by solving $k$ one-dimensional problems (using Equation (1)), one for each good.

We now state the basic properties from Hart and Nisan [2017, Propositions 5 and 6] needed for our proof.

Proposition 1. (i) Let $\mu=(q, s)$ be a mechanism for $k$ goods with buyer payoff function $b$. Then $\mu=(q, s)$ satisfies IC if and only if $b$ is a convex function and for all $x$ the vector $q(x)$ is a subgradient of $b$ at $x$ (i.e., $b(\tilde{x})-b(x) \geq q(x) \cdot(\tilde{x}-x)$ for all $\tilde{x})$.

(ii) $\operatorname{REV}(X)=\sup _{\mu} R(\mu ; X)$ with the supremum taken over all IC, IR, and NPT mechanisms $\mu$.

\subsection{Distributions}

As we show formally in Appendix A.1, for the results of the present article, we can limit ourselves without loss of generality to valuations that admit a density function (this follows from general continuity properties of the revenue, which we prove in Appendix A, and are of independent interest).

\footnotetext{
${ }^{9}$ When the goods are infinitely divisible and the valuations are linear in quantities, $q_{i}$ may be alternatively viewed as the quantity of good $i$ that the buyer gets.

${ }^{10}$ The scalar product of two $n$-dimensional vectors $y=\left(y_{1}, \ldots, y_{n}\right)$ and $z=\left(z_{1}, \ldots, z_{n}\right)$ is $y \cdot z=\sum_{i=1}^{n} y_{i} z_{i}$.

${ }^{11}$ Individual rationality recognizes that, regardless of his valuation, the buyer can obtain an expected payoff of zero by not participating in the mechanism.
} 
In what follows, we thus assume that every nonnegative random variable $X$ has an absolutely continuous cumulative distribution function, $F(t)=\mathbb{P}[X \leq t]=\mathbb{P}[X<t]$, with an associated density function $f(t)$. We denote by $G$ the tail probability, i.e.,

$$
G(t):=1-F(t)=\int_{t}^{\infty} f(u) \mathrm{d} u=\mathbb{P}[X \geq t],
$$

and by $H$ the cumulative tail probability, i.e.,

$$
H(t):=\int_{0}^{t} G(u) \mathrm{d} u=\mathbb{E}[\min \{X, t\}]
$$

(the equality holds, since $\mathbb{E}[\min \{X, t\}]=\int_{0}^{\infty} \mathbb{P}[\min \{X, t\} \geq u] \mathrm{d} u=\int_{0}^{t} \mathbb{P}[X \geq u] \mathrm{d} u=$ $\left.\int_{0}^{t} G(u) \mathrm{d} u\right)$.

Let $r:=\operatorname{Rev}(X)>0$ be $^{12}$ the optimal revenue from $X$; then Equation (1) implies $G(t) \leq r / t$, which together with $G(t) \leq 1$ gives

$$
G(t) \leq \min \left\{\frac{r}{t}, 1\right\}
$$

Therefore,

$$
H(t) \leq \int_{0}^{r} 1 \mathrm{~d} u+\int_{r}^{t} \frac{r}{u} \mathrm{~d} u=r+r \log \frac{t}{r},
$$

for every $t \geq r$ (and $H(t) \leq t$ for $t \leq r)$.

\subsection{Change of Units}

We start with a trivial, but useful, change of units. For every $0<\lambda_{1}, \ldots, \lambda_{k} \leq 1$, let $\mathcal{M}_{\lambda_{1}, \ldots, \lambda_{k}}$ denote the set of all IC and IR mechanisms $\mu=(q, s)$ that satisfy $q_{i}(x) \in\left[0, \lambda_{i}\right]$ (instead of $q_{i}(x) \in$ $[0,1])$ for every $x \in \mathbb{R}_{+}^{k}$ and $i=1, \ldots, k$. The set of all IC and IR mechanisms, which we denote by $\mathcal{M}$, is thus the same as $\mathcal{M}_{1, \ldots, 1}$.

Lemma 2. For every $0<\lambda_{1}, \ldots, \lambda_{k} \leq 1$, we have

$$
\operatorname{REV}\left(X_{1}, \ldots, X_{k}\right)=\sup _{\mu \in \mathcal{M}_{\lambda_{1}, \ldots, \lambda_{k}}} R\left(\mu ; \tilde{X}_{1}, \ldots, \tilde{X}_{k}\right),
$$

where $\tilde{X}_{i}:=\left(1 / \lambda_{i}\right) X_{i}$ for $i=1, \ldots, k$.

Proof. Given $\tilde{\mu}=(\tilde{q}, \tilde{s})$ with $\tilde{q}_{i}(x) \in\left[0, \lambda_{i}\right]$ for all $i$, define $\mu=(q, s)$ by $q_{i}\left(x_{1}, \ldots, x_{k}\right):=$ $\left(1 / \lambda_{i}\right) \tilde{q}_{i}\left(x_{1} / \lambda_{1}, \ldots, x_{k} / \lambda_{k}\right) \in[0,1]$ and $s\left(x_{1}, \ldots, x_{k}\right):=\tilde{s}\left(x_{1} / \lambda_{1}, \ldots, x_{k} / \lambda_{k}\right)$ (and thus $b\left(x_{1}, \ldots, x_{k}\right)=\tilde{b}\left(x_{1} / \lambda_{1}, \ldots, x_{k} / \lambda_{k}\right)$ for the corresponding buyer's payoff functions). It is immediate to see that $\mu$ is IC and IR if and only if $\tilde{\mu}$ is IC and IR, and that $\mathbb{E}\left[\tilde{s}\left(\tilde{X}_{1}, \ldots, \tilde{X}_{k}\right)\right]=\mathbb{E}\left[s\left(X_{1}, \ldots, X_{k}\right)\right]$. Conversely, given $\mu$ one generates $\tilde{\mu}$ by the reverse transformation.

\section{OVERVIEW OF THE PROOF}

The first part of the proof is similar to the proofs of Theorems A and B in Hart and Nisan [2017 $]^{13}$ except that, where they split the buyer's space of values $\left(x_{1}, x_{2}\right) \in \mathbb{R}_{+}^{2}$ in half along the diagonal $x_{1}=x_{2}$, we split the space into two regions $x_{1} \geq \lambda x_{2}$ and $x_{1}<\lambda x_{2}$ along a possibly nonsymmetric diagonal $x_{1}=\lambda x_{2}$ (the precise value of $\lambda$ will be chosen later). For any two-good mechanism, the

\footnotetext{
${ }^{12}$ The continuity of $F$ implies that $X$ cannot be identically zero, and so the optimal revenue $\operatorname{Rev}(X)$ must be positive (just sell the good at a small enough positive price).

${ }^{13}$ The reader is encouraged to look at these proofs and the explanations there.
} 
revenue in each of the two regions can be estimated by constructing from it appropriate one-good mechanisms, which eventually leads to a key bound: see Proposition 4 in Section 4. (Rather than working directly with the two asymmetric regions, which is cumbersome, the proof simplifies computations by first making an appropriate change of units, which amounts to rescaling the probabilities that the goods are received: see Lemma 2 in Section 2.3.) Once we have the bound given in Proposition 4, we need to estimate the maximum of a certain integral expression-which is essentially the additional revenue that is achievable beyond the separate one-good revenues - over pairs of nonnegative functions $\varphi_{1}, \varphi_{2}$ whose sum $\varphi_{1}+\varphi_{2}$ is nondecreasing. This is accomplished in Proposition 5, by considering the appropriate extreme functions and then carefully estimating the relevant terms (this is the hardest part of the proof). In Section 6, we put everything together, and, by choosing the best possible $\lambda$ (specifically, $\lambda=1 / \sqrt{e}$ ), prove the $62 \%$ bound (Theorem 7 ). Then in Section 7, we show the $73 \%$ bound for Myerson-regular goods (Theorem 9), and then we also deal with monotonic mechanisms. There are two appendices: Appendix A establishes that, under quite permissive conditions, the seller's revenue is continuous in the distribution of the buyer's valuation, a result that we use in our proof, but that is also of independent interest, and Appendix B provides a simple illustration of how the "nonsymmetric diagonal" construct alone can produce useful bounds.

\section{BOUNDING THE REVENUE BY NONSYMMETRIC DECOMPOSITION}

This section provides the basic decomposition with respect to a nonsymmetric diagonal (equivalently, we make a corresponding change of units and use the symmetric diagonal; see Section 2.3).

Given a two-good random valuation $\left(X_{1}, X_{2}\right)$, for $i=1,2$ let $F_{i}$ denote the cumulative distribution function of $X_{i}$, and let $f_{i}, G_{i}$, and $H_{i}$ be the associated funtions as defined in Section 2.2 (namely, the density, tail probability, and cumulative tail probability functions, respectively). We let $r_{i}:=\operatorname{Rev}\left(X_{i}\right)$ be the optimal revenue that can be obtained from good $i$, and define two useful auxiliary functions $K_{1}$ and $K_{2}$ :

$$
\begin{aligned}
& K_{1}(t):=f_{2}(t)\left(H_{1}(t)-r_{1}\right)-G_{1}(t) G_{2}(t), \\
& K_{2}(t):=f_{1}(t)\left(H_{2}(t)-r_{2}\right)-G_{1}(t) G_{2}(t) .
\end{aligned}
$$

The $K$ functions arise in our bound in Proposition 4, and are crucial to our analysis; they may be viewed as a kind of "joint virtual valuation" (see Equation (15), the decomposition (23), and Lemma 8 in Section 7).

The following lemma, which slightly generalizes Lemma 19 in Hart and Nisan [2017] (it replaces the factor $1-q\left(x_{0}\right)$ there with $\lambda-q\left(x_{0}\right)$ here), obtains a better bound on the revenue of a mechanism by "rescaling" its allocation function $q$ so it covers the entire interval $[0, \lambda]$.

LEMMA 3. Let $X$ be a one-good random valuation with values bounded from below by some $x_{0} \geq 0$. Then for every IC mechanism $\mu=(q, s)$ that satisfies $q(x) \leq \lambda$ for all ${ }^{14} x \geq x_{0}$, we have

$$
R(\mu ; X) \leq\left(\lambda-q\left(x_{0}\right)\right) \operatorname{Rev}(X)+s\left(x_{0}\right) .
$$

Proof. The function $q$ is nondecreasing (because $q$ is the derivative of the buyer's payoff function $b$, which is convex), and so $q\left(x_{0}\right) \leq q(x) \leq \lambda$ for all ${ }^{15} x \geq x_{0}$.

If $q\left(x_{0}\right)=\lambda$, then $q(x)=q\left(x_{0}\right)=\lambda$ for all $x \geq x_{0}$, hence $s(x)=s\left(x_{0}\right)$ for all $x \geq x_{0}$ by IC; therefore, $\mathbb{E}[s(X)]=s\left(x_{0}\right)$ and Equation (6) holds as equality.

\footnotetext{
${ }^{14}$ It suffices to require $q(x) \leq \lambda$ for $x$ in the support of $X$. As in Hart and Reny [2015], one can always extend a $k$-good mechanism to the whole space $\mathbb{R}_{+}^{k}$ without increasing its menu beyond taking closure, and so the bound extends to all $\mathbb{R}_{+}^{k}$.

${ }^{15}$ If the values of $X$ are bounded from above by some finite $x_{1}$, then we can replace $\lambda$ with $q\left(x_{1}\right)$.
} 
If $q\left(x_{0}\right)<\lambda$, then define a new mechanism $\hat{\mu}=(\hat{q}, \hat{s})$ by $\hat{q}(x):=\theta\left(q(x)-q\left(x_{0}\right)\right)$ and $\hat{s}(x):=$ $\theta\left(s(x)-s\left(x_{0}\right)\right)$, and thus $\hat{b}(x):=\theta\left(b(x)-\left(x-x_{0}\right) q\left(x_{0}\right)-b\left(x_{0}\right)\right)$, where $\theta:=1 /\left(\lambda-q\left(x_{0}\right)\right)>0$ (so that $0 \leq \hat{q}(x) \leq 1)$. It is immediate to verify that $(\hat{q}, \hat{s})$ is an IC and IR mechanism: indeed, $[\hat{q}(x) \cdot x-\hat{s}(x)]-\left[\hat{q}\left(x^{\prime}\right) \cdot x-\hat{s}\left(x^{\prime}\right)\right]=\theta\left([q(x) \cdot x-s(x)]-\left[q\left(x^{\prime}\right) \cdot x-s\left(x^{\prime}\right)\right]\right) \geq 0$, and $\hat{b}\left(x_{0}\right)=$ 0 . Therefore, $\operatorname{Rev}(X) \geq \mathbb{E}[\hat{s}(X)]=\theta\left(\mathbb{E}[s(X)]-s\left(x_{0}\right)\right)$, which yields Equation (6).

We now come to the main result of this section, which generalizes the decomposition of the proofs of Theorems A and B in Hart and Nisan [2017]: the revenue from two goods is bounded by the sum of the separate one-good revenues and an additional term ("the $K$-term"), which will be estimated in the next section.

Proposition 4. Let $X=\left(X_{1}, X_{2}\right)$ be a two-good random valuation with independent goods (i.e., $X_{1}$ and $X_{2}$ are independent nonnegative real random variables), and let $\mu=(q, s)$ be a two-good $I C, I R$, and NPT mechanism that satisfies $q_{i}(x) \leq \lambda_{i}$ for all $x \in \mathbb{R}_{+}^{2}$ and $i=1,2$. Then the functions $\varphi_{i}: \mathbb{R}_{+} \rightarrow\left[0, \lambda_{i}\right]$ given by $\varphi_{i}(t):=q_{i}(t, t)$ for $i=1,2$ are such that $\varphi_{1}+\varphi_{2}$ is nondecreasing and

$$
R\left(\mu ; X_{1}, X_{2}\right) \leq \lambda_{1} r_{1}+\lambda_{2} r_{2}+\int_{0}^{\infty}\left(\varphi_{1}(t) K_{1}(t)+\varphi_{2}(t) K_{2}(t)\right) \mathrm{d} t .
$$

Proof. The first part of the proof, which yields Equation (10), follows the same lines as the proof of Theorem B in Appendix A.1 of Hart and Nisan [2017], but with the appropriate modifications, because here $X_{1}$ and $X_{2}$ are not identically distributed, the mechanism $\mu$ is not symmetric, and each $q_{i}$ is bounded by $\lambda_{i}$.

We will write $Y$ for $X_{1}$ and $Z$ for $X_{2}$, and so $X=(Y, Z)$.

For every $t \geq 0$ define ${ }^{16} \Phi(t):=b(t, t)$ and $\varphi_{i}(t):=q_{i}(t, t)$. By Proposition 1(i) the function $\Phi$ is convex and $q(t, t)=\left(\varphi_{1}(t), \varphi_{2}(t)\right)$ is a subgradient of $b$ at $(t, t)$, and so $\varphi_{1}(t)+\varphi_{2}(t)$ is a subgradient of $\Phi$ at $t$. Therefore, $\varphi_{1}+\varphi_{2}$ is a nondecreasing function, and $\Phi(u)=\int_{0}^{u}\left(\varphi_{1}(t)+\varphi_{2}(t)\right) \mathrm{d} t$ (use Corollary 24.2.1 in Rockafellar [1970], recalling that $\Phi(0)=b(0,0)=0$ by NPT).

Consider first the region $Y \geq Z$. For each fixed value $z \geq 0$ of the second good such that $\mathbb{P}[Y \geq z]>0$, define from the two-good mechanism $\mu=(q, s)$ a one-good mechanism $\mu^{z}=$ $\left(q^{z}, s^{z}\right)$ for the first good by replacing the allocation of the second good with an equivalent decrease in payment; that is, the allocation of the first good is unchanged, i.e., $q^{z}(y):=q_{1}(y, z)$, and the payment is $s^{z}(y):=s(y, z)-z q_{2}(y, z)$, for every $y \geq 0$; note that the buyer's payoff remains the same: $b^{z}(y)=b(y, z)$. The mechanism $\mu^{z}$ is IC and IR for $y$, since $\mu$ is IC and IR for $(y, z)$. Let $Y^{z}$ denote the random variable $Y$ conditional on the event $Y \geq z$, and consider the revenue $R\left(\mu^{z} ; Y^{z}\right)=\mathbb{E}\left[s^{z}\left(Y^{z}\right)\right]=\mathbb{E}\left[s^{z}(Y) \mid Y \geq z\right]$ of $\mu^{z}$ from $Y^{z}$. We have $Y^{z} \geq z, q^{z}(z)=$ $q_{1}(z, z)=\varphi_{1}(z)$, and $s^{z}(z)=s(z, z)-z q_{2}(z, z)=z q_{1}(z, z)-b(z, z)=z \varphi_{1}(z)-\Phi(z)$, and so, applying Lemma 3 above to $Y^{z}$, we have

$$
\mathbb{E}\left[s^{z}(Y) \mid Y \geq z\right] \leq\left(\lambda_{1}-\varphi_{1}(z)\right) \operatorname{Rev}\left(Y^{z}\right)+z \varphi_{1}(z)-\Phi(z) .
$$

Now $\operatorname{Rev}\left(Y^{z}\right) \leq \operatorname{Rev}(Y) / \mathbb{P}[Y \geq z]$ (use Equation (1): any posted-price mechanism for $Y^{z}$ yields, when applied to $Y$, at least $\mathbb{P}[Y \geq z]$ times the revenue from $\left.Y^{z}\right)$. Substituting this into Equation (8) and multiplying by $\mathbb{P}[Y \geq z]$ gives

$$
\mathbb{E}\left[s^{z}(Y) \mathbf{1}_{Y \geq z}\right] \leq\left(\lambda_{1}-\varphi_{1}(z)\right) r_{1}+\left(z \varphi_{1}(z)-\Phi(z)\right) \mathbb{P}[Y \geq z]
$$

for all $z \geq 0$ (which trivially includes those $z$ where $\mathbb{P}[Y \geq z]=0$ ). Taking expectation over the values $z$ of $Z$ yields

$$
\mathbb{E}\left[s^{Z}(Y) \mathbf{1}_{Y \geq Z}\right] \leq \lambda_{1} r_{1}-r_{1} \mathbb{E}\left[\varphi_{1}(Z)\right]+\mathbb{E}\left[\left(Z \varphi_{1}(Z)-\Phi(Z) \mathbf{1}_{Y \geq Z}\right] .\right.
$$

\footnotetext{
${ }^{16}$ Notice that $\Phi$ here is $2 \Phi$ in Hart and Nisan [2017].
} 
For $y \geq z \geq 0$, we have $s(y, z)=s^{z}(y)+z q_{2}(y, z) \leq s^{z}(y)+z q_{2}(y, y)=s^{z}(y)+z \varphi_{2}(y)$ (by the monotonicity of $q_{2}$ in its second variable, again by the convexity of $b$ ), which together with Equation (9) yields

$$
\begin{aligned}
\mathbb{E}\left[s(Y, Z) \mathbf{1}_{Y \geq Z}\right] & \leq \mathbb{E}\left[s^{Z}(Y) \mathbf{1}_{Y \geq Z}\right]+\mathbb{E}\left[Z \varphi_{2}(Y) \mathbf{1}_{Y \geq Z}\right] \\
& \leq \lambda_{1} r_{1}-r_{1} \mathbb{E}\left[\varphi_{1}(Z)\right]+\mathbb{E}\left[\left(Z \varphi_{2}(Y)+Z \varphi_{1}(Z)-\Phi(Z)\right) \mathbf{1}_{Y \geq Z}\right] \\
& =\lambda_{1} r_{1}-r_{1} \mathbb{E}\left[\varphi_{1}(Z)\right]+\mathbb{E}\left[\left(\Lambda \varphi_{2}(Y)+\Lambda \varphi_{1}(Z)-\Phi(\Lambda)\right) \mathbf{1}_{Y \geq Z}\right],
\end{aligned}
$$

where we put $\Lambda:=\min \{Y, Z\}$.

Consider next the $Z>Y$. Interchanging $\mathrm{Y}$ and $Z$ and using $Z>y$ instead of $Z \geq y$ throughout gives

$$
\mathbb{E}\left[s(Y, Z) \mathbf{1}_{Z>Y}\right] \leq \lambda_{2} r_{2}-r_{2} \mathbb{E}\left[\varphi_{2}(Y)\right]+\mathbb{E}\left[\left(\Lambda \varphi_{1}(Z)+\Lambda \varphi_{2}(Y)-\Phi(\Lambda)\right) \mathbf{1}_{Z>Y}\right] .
$$

Adding the last two inequalities yields

$$
\begin{aligned}
\mathbb{E}[s(Y, Z)] \leq & \lambda_{1} r_{1}+\lambda_{2} r_{2}-r_{1} \mathbb{E}\left[\varphi_{1}(Z)\right]-r_{2} \mathbb{E}\left[\varphi_{2}(Y)\right] \\
& +\mathbb{E}\left[\Lambda \varphi_{1}(Z)+\Lambda \varphi_{2}(Y)-\Phi(\Lambda)\right] \\
= & \lambda_{1} r_{1}+\lambda_{2} r_{2} \\
& +\mathbb{E}\left[\varphi_{1}(Z)\left(\Lambda-r_{1}\right)\right]+\mathbb{E}\left[\varphi_{2}(Y)\left(\Lambda-r_{2}\right)\right]-\mathbb{E}[\Phi(\Lambda)] .
\end{aligned}
$$

Now, we have

$$
\begin{aligned}
\mathbb{E}\left[\varphi_{1}(Z)\left(\Lambda-r_{1}\right)\right] & =\int_{0}^{\infty} \varphi_{1}(z)\left(\mathbb{E}[\min \{Y, z\}]-r_{1}\right) f_{2}(z) \mathrm{d} z \\
& =\int_{0}^{\infty} \varphi_{1}(z)\left(H_{1}(z)-r_{1}\right) f_{2}(z) \mathrm{d} z
\end{aligned}
$$

(use $\Lambda=\min \{Y, Z\}$ and Equation (2)). Similarly,

$$
\mathbb{E}\left[\varphi_{2}(Y)\left(\Lambda-r_{2}\right)\right]=\int_{0}^{\infty} \varphi_{2}(y)\left(H_{2}(y)-r_{2}\right) f_{1}(y) \mathrm{d} y .
$$

Let $F_{\Lambda}$ be the cumulative distribution function of $\Lambda=\min \{Y, Z\}$; then $1-F_{\Lambda}(u)=G_{\Lambda}(u)=$ $G_{1}(u) G_{2}(u)$, and

$$
\begin{aligned}
\mathbb{E}[\Phi(\Lambda)] & =\int_{0}^{\infty} \Phi(u) \mathrm{d} F_{\Lambda}(u)=-\int_{0}^{\infty} \Phi(u) \mathrm{d} G_{\Lambda}(u) \\
& =\left[-\Phi(u) G_{\Lambda}(u)\right]_{0}^{\infty}+\int_{0}^{\infty} \Phi^{\prime}(u) G_{\Lambda}(u) \mathrm{d} u \\
& =\int_{0}^{\infty} \Phi^{\prime}(u) G_{\Lambda}(u) \mathrm{d} u \\
& =\int_{0}^{\infty}\left(\varphi_{1}(u)+\varphi_{2}(u)\right) G_{1}(u) G_{2}(u) \mathrm{d} u,
\end{aligned}
$$

where we integrated by parts to get the second line, ${ }^{17}$ and then used $\Phi(0)=0$ and $\Phi(\infty) G_{\Lambda}(\infty)=0$ (because $0 \leq \Phi(u) G_{\Lambda}(u) \leq 2 u\left(r_{1} / u\right)\left(r_{2} / u\right) \rightarrow 0$ as $u \rightarrow \infty$, with $\Phi(u) \leq 2 u$ following from $\left.\Phi^{\prime}(u) \leq 2\right)$.

Substituting Equations (11)-(13) into Equation (10) yields the result.

\footnotetext{
${ }^{17}$ Formally, we integrate by parts on a finite interval $[0, M]$ and then let $M \rightarrow \infty$. The functions $G_{\Lambda}$ and $\Phi$ are absolutely continuous (because $G_{i}=1-F_{i}$ for $i=1,2$ are absolutely continuous and $G_{\Lambda}=G_{1} G_{2}$, and $\Phi$ is convex and continuous).
} 


\section{BOUNDING THE $K$-TERM}

In this section, we bound from above the term $\int\left(\varphi_{1} K_{1}+\varphi_{2} K_{2}\right)$ in Equation (7) over all possible functions $\varphi_{i}$, which take values in $\left[0, \lambda_{i}\right]$, and whose sum $\varphi_{1}+\varphi_{2}$ is nondecreasing. This term is linear in the $\varphi_{i}$, and so, if each $\varphi_{i}$ were nondecreasing, it would suffice to consider only the extreme functions that take the values 0 and $\lambda_{i}$ (because any nondecreasing function is an average of such functions; see the remark below). However, we only require the sum to be nondecreasing, which requires a more delicate analysis; see Proposition 5. This result is then applied to our specific functions $K_{1}$ and $K_{2}$ to get the bound in Proposition 6 (this constitutes the core of the proof).

From now on, we will assume without loss of generality that $\lambda_{1} \leq \lambda_{2}$, and so $0<\lambda_{1} \leq \lambda_{2} \leq 1$. Let $K_{1}, K_{2}: \mathbb{R}_{+} \rightarrow \mathbb{R}$ be two functions, and define

$$
I:=\sup _{\varphi_{1}, \varphi_{2}} \int_{0}^{\infty}\left(\varphi_{1}(t) K_{1}(t)+\varphi_{2}(t) K_{2}(t)\right) \mathrm{d} t
$$

where the supremum is taken over all functions $\varphi_{i}: \mathbb{R}_{+} \rightarrow\left[0, \lambda_{i}\right]$ such that $\varphi:=\varphi_{1}+\varphi_{2}$ is nondecreasing.

To estimate $I$, for any $0 \leq a \leq b \leq c \leq \infty$ define

$$
\begin{aligned}
I(a, b, c):= & \int_{a}^{b} \lambda_{1} \max \left\{K_{1}(t), K_{2}(t)\right\} \mathrm{d} t \\
& +\int_{b}^{c}\left(\left(\lambda_{2}-\lambda_{1}\right) K_{2}(t)+\lambda_{1} \max \left\{K_{1}(t), K_{2}(t)\right\}\right) \mathrm{d} t \\
& +\int_{c}^{\infty}\left(\lambda_{1} K_{1}(t)+\lambda_{2} K_{2}(t)\right) \mathrm{d} t \\
= & \lambda_{1} \int_{a}^{c} \max \left\{K_{1}(t), K_{2}(t)\right\} \mathrm{d} t+\lambda_{1} \int_{c}^{\infty}\left(K_{1}(t)+K_{2}(t)\right) \mathrm{d} t \\
& +\left(\lambda_{2}-\lambda_{1}\right) \int_{b}^{\infty} K_{2}(t) \mathrm{d} t .
\end{aligned}
$$

It is immediate to see that $I(a, b, c)$ is nothing other than $\int\left(\varphi_{1} K_{1}+\varphi_{2} K_{2}\right)$ for the following functions $\varphi_{1}$ and $\varphi_{2}$ :

\begin{tabular}{|c||c|c|c|}
\hline & $a \leq t<b$ & $b \leq t<c$ & $t \geq c$ \\
\hline \hline$\varphi_{1}(t)$ & $\lambda_{1} \mathbf{1}_{K_{1}(t) \geq K_{2}(t)}$ & $\lambda_{1} \mathbf{1}_{K_{1}(t) \geq K_{2}(t)}$ & $\lambda_{1}$ \\
\hline$\varphi_{2}(t)$ & $\lambda_{1} \mathbf{1}_{K_{1}(t)<K_{2}(t)}$ & $\lambda_{1} \mathbf{1}_{K_{1}(t)<K_{2}(t)}+\lambda_{2}-\lambda_{1}$ & $\lambda_{2}$ \\
\hline
\end{tabular}

Their sum $\varphi_{1}+\varphi_{2}$ then equals

\begin{tabular}{|c||c|c|c|}
\hline & $a \leq t<b$ & $b \leq t<c$ & $t \geq c$, \\
\hline \hline$\varphi_{1}(t)+\varphi_{2}(t)$ & $\lambda_{1}$ & $\lambda_{2}$ & $\lambda_{1}+\lambda_{2}$ \\
\hline
\end{tabular}

which is a nondecreasing function, and so $I(a, b, c) \leq I$.

Proposition 5. Suppose that $0<\lambda_{1} \leq \lambda_{2} \leq 1$. Then

$$
I=\sup _{0 \leq a \leq b \leq c \leq \infty} I(a, b, c) .
$$

Remark. We will use the following well-known result. Every nondecreasing function $\psi:[u, v] \rightarrow[0,1]$ (where $-\infty \leq u \leq v \leq \infty$ ) can be expressed as an (integral) average of 
nondecreasing functions that take only the values $0 \mathrm{and}^{18} 1$. More generally, every nondecreasing function $\psi:[u, v] \rightarrow[\alpha, \beta]$ (where $\alpha \leq \beta$ are finite) can be expressed as an average of nondecreasing functions that take only the two values $\alpha$ and $\beta$ (when $\alpha<\beta$, apply the above to $(\psi-\alpha) /(\beta-\alpha)$, which takes values in $[0,1])$. Therefore, when we maximize a linear functional $\int_{u}^{v} \psi(t) K(t) \mathrm{d} t$ over all nondecreasing functions $\psi:[u, v] \rightarrow[\alpha, \beta]$, it suffices to consider those functions that take only the two extreme values $\alpha$ and $\beta$.

Proof. We have seen above that $I \geq I(a, b, c)$ for every $a \leq b \leq c$. We now show that $I \leq$ $\sup _{a \leq b \leq c} I(a, b, c)$.

The proof proceeds as follows: We partition the range of $\varphi$ into three intervals: $\left[0, \lambda_{1}\right],\left(\lambda_{1}, \lambda_{2}\right]$, and $\left(\lambda_{2}, \lambda_{1}+\lambda_{2}\right]$; provide simple pointwise bounds on $\varphi_{1}(t) K_{1}(t)+\varphi_{2}(t) K_{2}(t)$ in each interval; as these bounds are affine in $\varphi$, we apply the remark above.

For each $t$, given $\varphi(t)=\varphi_{1}(t)+\varphi_{2}(t)$, the expression $\varphi_{1}(t) K_{1}(t)+\varphi_{2}(t) K_{2}(t)$ is maximized by putting as much weight as possible-subject to the constraints $0 \leq \varphi_{i}(t) \leq \lambda_{i}$-on the higher of $K_{1}(t)$ and $K_{2}(t)$. This gives the following upper bounds on $\varphi_{1}(t) K_{1}(t)+\varphi_{2}(t) K_{2}(t)$ :

- $\varphi(t) \max \left\{K_{1}(t), K_{2}(t)\right\}$ for every $t$ in the interval where $0 \leq \varphi(t) \leq \lambda_{1}$;

- $\left(\varphi(t)-\lambda_{1}\right) K_{2}(t)+\lambda_{1} \max \left\{K_{1}(t), K_{2}(t)\right\}$ for every $t$ in the interval where $\lambda_{1} \leq \varphi(t) \leq \lambda_{2}$ (because $\varphi_{1}(t) \leq \lambda_{1}$ implies $\left.\varphi_{2}(t) \geq \varphi(t)-\lambda_{1}\right)$; and

- $\left(\varphi(t)-\lambda_{2}\right) K_{1}(t)+\left(\varphi(t)-\lambda_{1}\right) K_{2}(t)+\left(\lambda_{1}+\lambda_{2}-\varphi(t)\right) \max \left\{K_{1}(t), K_{2}(t)\right\}$ for every $t$ in the interval where $\lambda_{2} \leq \varphi(t) \leq \lambda_{1}+\lambda_{2}$.

In each one of these three intervals the bound is affine in $\varphi$ and so, by the remark above, when maximizing over nondecreasing $\varphi$, it suffices to consider solely those functions $\varphi$ that take only the corresponding two extreme values. Altogether, such a $\varphi$ takes only the values $0, \lambda_{1}, \lambda_{2}$, and $\lambda_{1}+\lambda_{2}$, say on the intervals $(0, a),(a, b),(b, c)$, and $(c, \infty)$, respectively-and then $\int\left(\varphi_{1} K_{1}+\varphi_{2} K_{2}\right)$ becomes precisely $I(a, b, c)$. Thus, indeed $I \leq \sup I(a, b, c)$.

We now come to the main argument of our proof, which yields, using Proposition 5, an upper bound on the $K$-term for our specific functions $K_{1}$ and $K_{2}$.

Proposition 6. Let $0<\lambda_{1} \leq \lambda_{2} \leq 1$, and let $K_{1}, K_{2}$ be given by Equations (4) and (5). Then

$$
I \leq \frac{1}{e}\left(\lambda_{2} r_{1}+\lambda_{1} r_{2}+\lambda_{1}(e-1) \min \left\{r_{1}, r_{2}\right\}\right) .
$$

Proof. Recalling Equation (2), we have the following: for each $i=1,2$, the function $H_{i}(t)$ is continuous and strictly increasing at each $t$ in the support of $X_{i}$ (because $G_{i}(t)>0$ there), and $H_{i}(\infty)=\mathbb{E}\left[X_{i}\right] \geq r_{i}$ (with strict inequality unless $X_{i}$ is constant, in which case everything trivializes). Therefore, there exists a finite $\tau_{i}$ such that $H_{i}\left(\tau_{i}\right)=r_{i}$; since for all $t<r_{i}$, we have

\footnotetext{
${ }^{18}$ Assume first that $\psi(v)=1$. If $\psi$ is a right-continuous function, then $\psi$ may be viewed as a cumulative distribution function on $[u, v]$, and we have $\psi(t)=\int_{[u, t]} \mathrm{d} \psi(x)=\int_{[u, v]} 1_{[x, v]}(t) \mathrm{d} \psi(x)$ for every $t \in[u, v]$ (where $1_{E}$ is the indicator function of the set $E$, i.e., $\mathbf{1}_{E}(t)=1$ if $t \in E$ and $\mathbf{1}_{E}(t)=0$ otherwise). If $\psi$ is not necessarily right-continuous, then let $\psi_{+}(t):=\lim _{t^{\prime} \backslash t} \psi\left(t^{\prime}\right)$ (which is right-contiuous), $\psi_{-}(t):=\lim _{t^{\prime}} \nearrow t \psi\left(t^{\prime}\right)$, and take $\lambda_{t} \in[0,1]$ such that $\psi(t)=$ $\lambda_{t} \psi_{+}(t)+\left(1-\lambda_{t}\right) \psi_{-}(t)$; then $\psi=\int_{[u, v]}\left(\lambda_{x} 1_{[x, v]}+\left(1-\lambda_{x}\right) 1_{(x, v]}\right) \mathrm{d} \psi_{+}(x)$.

If $0<\psi(v)<1$, then $\psi=\psi(v) \tilde{\psi}+(1-\psi(v)) 0$, where $\tilde{\psi}(t):=\psi(t) / \psi(v)$ and 0 is the zero function (i.e., $0(t)=0$ for all $t$ ), and we apply the above to $\tilde{\psi}$. Finally, if $\psi(v)=0$, then $\psi=0$.

As an application, every one-good IC and IR mechanism $\mu=(q, s)$ can be expressed as an average of posted-price mechanisms $\mu_{p}=\left(q_{p}, s_{p}\right)$, where $q_{p}=1_{[p, \infty)}$ and $s_{p}=p 1_{[p, \infty)}$. Indeed, $q$ is nondecreasing, and taking it to be, say, right-continuous (which corresponds to seller-favorability), we have $q=\int q_{p} \mathrm{~d} q(p)$, and thus $s=\int s_{p} \mathrm{~d} q(p)$, and $b=$ $\int b_{p} \mathrm{~d} q(p)$ for the corresponding buyer payoff functions (this decomposition provides a proof of Equation (1)).
} 
$H_{i}(t) \leq t<r_{i}$ (because $\left.G_{i} \leq 1\right)$, it follows that

$$
\tau_{i} \geq r_{i}
$$

Put $L_{i}(t):=G_{j}(t)\left(H_{i}(t)-r_{i}\right)$; taking derivatives gives

$$
L_{i}^{\prime}(t)=-f_{j}(t)\left(H_{i}(t)-r_{i}\right)+G_{j}(t) G_{i}(t)=-K_{i}(t) .
$$

We will use the following estimates:

$$
\int_{u}^{\infty} G_{1}(t) G_{2}(t) \mathrm{d} t \leq \int_{u}^{\infty} \frac{r_{1}}{t} \frac{r_{2}}{t} \mathrm{~d} t=\frac{r_{1} r_{2}}{u}
$$

for every $u>0$ (because $\left.G_{i}(t) \leq r_{i} / t\right)$;

$$
H_{i}(u)-r_{i} \leq r_{i} \log \frac{u}{r_{i}}
$$

for every $u \geq r_{i}$ (recall Equation (3)); and, thus,

$$
L_{i}(u)=G_{j}(u)\left(H_{i}(u)-r_{i}\right) \leq \frac{r_{i} r_{j}}{u} \log \frac{u}{r_{i}}
$$

for every $u \geq r_{i}$. The last inequality implies that $L_{i}(u) \rightarrow 0$ as $u \rightarrow \infty$, and so

$$
\int_{u}^{\infty} K_{i}(t) \mathrm{d} t=\left[-L_{i}(t)\right]_{u}^{\infty}=L_{i}(u)
$$

Finally, letting $\{i, j\}=\{1,2\}$, we have

$$
\begin{aligned}
L_{i}(u) & \leq \frac{1}{e} r_{j} \text { and } \\
L_{i}(u)+\frac{r_{i} r_{j}}{u} & \leq r_{j}
\end{aligned}
$$

for every $u \geq r_{i}$ (use Equation (17) together with $\log x / x \leq 1 / e$ and $(\log x+1) / x \leq 1$ for all $x>0$; note that there is no typo here: these bounds on $L_{i}$ use $r_{j}$ rather than $r_{i}$ ).

We need to bound $I(a, b, c)$. For the last term of Equation (14), we have, by Equations (18) and (19),

$$
\int_{b}^{\infty} K_{2}(t) \mathrm{d} t \leq \frac{1}{e} r_{1}
$$

and so it remains to estimate $J(a, c):=\int_{a}^{c} \max \left\{K_{1}, K_{2}\right\}+\int_{c}^{\infty}\left(K_{1}+K_{2}\right)$. A main difficulty in doing so is that the $K_{i}$ are neither nonnegative nor monotonic, and may change signs many times. To handle this, we define for each $i$ an auxiliary function $M_{i}(t):=f_{j}(t)\left(H_{i}(t)-r_{i}\right)=K_{i}(t)+G_{1}(t) G_{2}(t)$, which vanishes at $t=\tau_{i}$, is nonpositive before $\tau_{i}$, and nonnegative after $\tau_{i}$; i.e., $M_{i}(t) \geq 0$ for $t \geq \tau_{i}$ and $M_{i}(t) \leq 0$ for $t \leq \tau_{i}$.

We distinguish three cases according to the location of $a$ relative to $\tau_{1}$ and $\tau_{2}$ (the points where $M_{1}$ and $M_{2}$ change sign); without loss of generality ${ }^{19}$ assume that $\tau_{1} \leq \tau_{2}$.

- Case 1. $a \geq \max \left\{\tau_{1}, \tau_{2}\right\}=\tau_{2}$. For every $t \geq a$, we have $M_{i}(t) \geq 0$ (because $\left.t \geq a \geq \tau_{i}\right)$, and thus ${ }^{20}$

$$
\begin{aligned}
\max \left\{K_{1}(t), K_{2}(t)\right\} & =\max \left\{M_{1}(t), M_{2}(t)\right\}-G_{1}(t) G_{2}(t) \\
& \leq M_{1}(t)+M_{2}(t)-G_{1}(t) G_{2}(t) \\
& =K_{1}(t)+K_{2}(t)+G_{1}(t) G_{2}(t) .
\end{aligned}
$$

\footnotetext{
${ }^{19}$ The expression $J(a, b)$ that we estimate now is symmetric in $i=1,2$, and so the assumption that $\lambda_{1} \leq \lambda_{2}$ is irrelevant here; we thus assume that $\tau_{1} \leq \tau_{2}$.

${ }^{20}$ This is the inequality $\max \{x, y\} \leq x+y+z$ whenever $x, y \geq-z$. As simple as it is, it turns out to be crucial in obtaining the overall better-than- $1 / 2$ bound.
} 
Since we clearly also have $K_{1}+K_{2} \leq K_{1}+K_{2}+G_{1} G_{2}$, we get

$$
\begin{aligned}
J(a, c) & \leq \int_{a}^{\infty}\left(K_{1}(t)+K_{2}(t)+G_{1}(t) G_{2}(t)\right) \mathrm{d} t \\
& \leq L_{1}(a)+L_{2}(a)+\frac{r_{1} r_{2}}{a}=: \bar{J}(a)
\end{aligned}
$$

by Equations (18) and (16). If, say, $r_{k} \leq r_{\ell}$ (where $\left.\{k, \ell\}=\{1,2\}\right)$ then using Equation (19) for $i=k$ and Equation (20) for $i=\ell$ (recall that $a \geq \tau_{i} \geq r_{i}$ for both $i$ ) yields ${ }^{21}$

$$
J(a, c) \leq \bar{J}(a) \leq \frac{1}{e} r_{\ell}+r_{k} .
$$

- Case 2. $\tau_{1} \leq a<\tau_{2}$. In the range $t \in\left[a, \tau_{2}\right) \subseteq\left[\tau_{1}, \tau_{2}\right)$, we have $M_{1}(t) \geq 0 \geq M_{2}(t)$, and so $K_{1}(t) \geq$ $K_{2}(t)$ and $K_{2}(t) \leq 0$; therefore, both $\max \left\{K_{1}(t), K_{2}(t)\right\}$ and $K_{1}(t)+K_{2}(t)$ are $\leq K_{1}(t)$, and thus, regardless of where $c$ is,

$$
\begin{aligned}
J(a, c) & \leq \int_{a}^{\tau_{2}} K_{1}(t) \mathrm{d} t+\bar{J}\left(\tau_{2}\right)=\left(L_{1}(a)-L_{1}\left(\tau_{2}\right)\right)+\left(L_{1}\left(\tau_{2}\right)+L_{2}\left(\tau_{2}\right)+\frac{r_{1} r_{2}}{\tau_{2}}\right) \\
& =L_{1}(a)+\frac{r_{1} r_{2}}{\tau_{2}} \leq \frac{1}{e} r_{2}+\min \left\{r_{1}, r_{2}\right\} \leq \frac{1}{e} r_{\ell}+r_{k},
\end{aligned}
$$

where we have used: $L_{2}\left(\tau_{2}\right)=0$ and $\tau_{2}=\max \left\{\tau_{1}, \tau_{2}\right\} \geq \max \left\{r_{1}, r_{2}\right\}$ (because $\tau_{i} \geq r_{i}$ ).

- Case 3. $a<\min \left\{\tau_{1}, \tau_{2}\right\}=\tau_{1}$. For every $t \leq \tau_{1}$, we have $K_{1}(t) \leq M_{1}(t) \leq 0$ and $K_{2}(t) \leq M_{2}(t) \leq 0$, and so both $\max \left\{K_{1}(t), K_{2}(t)\right\}$ and $K_{1}(t)+K_{2}(t)$ are $\leq 0$ in the interval [ $\left.a, \tau_{1}\right]$. Therefore, $J(a, c) \leq$ $J\left(\tau_{1}, \max \left\{c, \tau_{1}\right\}\right)$, to which we apply Case 2 (with $\left.a=\tau_{1}\right)$.

Thus, in all three cases the bound on $J(a, c)$ is $(1 / e) r_{\ell}+r_{k}=(1 / e)\left(r_{1}+r_{2}\right)+(1-$ $1 / e) \min \left\{r_{1}, r_{2}\right\}$; together with Equation (21), we get

$$
I(a, b, c) \leq \frac{\lambda_{1}}{e} r_{1}+\frac{\lambda_{1}}{e} r_{2}+\frac{\lambda_{1}(e-1)}{e} \min \left\{r_{1}, r_{2}\right\}+\frac{\lambda_{2}-\lambda_{1}}{e} r_{1},
$$

completing the proof.

Remarks. (a) If $\varphi_{1}$ and $\varphi_{2}$ are each required to be nondecreasing (rather than just their sum), then we get a smaller bound on $\int\left(\varphi_{1} K_{1}+\varphi_{2} K_{2}\right)$, namely:

$$
\begin{aligned}
& \sup _{\varphi_{1}, \varphi_{2}} \int_{0}^{\infty}\left(\varphi_{1}(t) K_{1}(t)+\varphi_{2}(t) K_{2}(t)\right) \mathrm{d} t \\
= & \sup _{0 \leq a \leq \infty} \lambda_{1} \int_{a}^{\infty} K_{1}(t) \mathrm{d} t+\sup _{0 \leq b \leq \infty} \lambda_{2} \int_{b}^{\infty} K_{2}(t) \mathrm{d} t \leq \frac{\lambda_{1}}{e} r_{2}+\frac{\lambda_{2}}{e} r_{1}
\end{aligned}
$$

(use the remark preceding the proof of Proposition 5 together with Equations (18) and (19)). Therefore, for mechanisms $\mu=(q, s)$ where $q_{1}(t, t)$ and $q_{2}(t, t)$ are monotonic-such as, for instance, symmetric mechanisms, where ${ }^{22} q_{1}(t, t)=q_{2}(t, t)$-we get, taking $\lambda_{1}=\lambda_{2}=1$ in Proposition 4 ,

$$
R(\mu ; X) \leq r_{1}+r_{2}+\frac{1}{e} r_{1}+\frac{1}{e} r_{2}=\left(1+\frac{1}{e}\right)\left(r_{1}+r_{2}\right) .
$$

This yields the bound $e /(e+1)$, which is better than $\sqrt{e} /(\sqrt{e}+1)$.

(b) If the functions $K_{i}$ satisfy a weak single-crossing property, i.e., if there exist $u_{i} \in[0, \infty]$ such that $K_{i}(t) \leq 0$ for $t<u_{i}$ and $K_{i}(t) \geq 0$ for $t>u_{i}$, then $\int_{0}^{\infty} \varphi_{i} K_{i} \leq \lambda_{i} \int_{u_{i}}^{\infty} K_{i}$. As in Remark (a)

\footnotetext{
${ }^{21}$ A slightly better estimate of $(2 / \sqrt{e}) \sqrt{r_{1} r_{2}}$ may be obtained here by directly maximizing $\bar{J}(a)$ over $a$; however, this will not improve the overall estimate, due to Cases 2 and 3.

${ }^{22}$ This proves Theorem B of Hart and Nisan [2017] for two independent and identically distributed goods.
} 
above, this yields the better bound $I \leq\left(\lambda_{1} / e\right) r_{2}+\left(\lambda_{2} / e\right) r_{1}$, and will be used when dealing with regular goods (see Lemma 8 and Theorem 9 in Section 7 below).

\section{COMPLETING THE PROOF}

Combining the results of the previous two sections yields the first part of our Main Result:

Theorem 7. Let $X=\left(X_{1}, X_{2}\right)$ be a two-good random valuation with independent goods. Then

$$
\frac{\operatorname{SREV}\left(X_{1}, X_{2}\right)}{\operatorname{REV}\left(X_{1}, X_{2}\right)} \geq \frac{\sqrt{e}}{\sqrt{e}+1} \approx 0.62
$$

Proof. Let ${ }^{23} R_{i}:=\operatorname{Rev}\left(X_{i}\right)$; thus $\operatorname{SRev}\left(X_{1}, X_{2}\right)=R_{1}+R_{2}$. Given $0<\lambda_{1} \leq \lambda_{2}$, put $\tilde{X}_{i}:=X_{i} / \lambda_{i}$ and $r_{i}:=\operatorname{Rev}\left(\tilde{X}_{i}\right)=R_{i} / \lambda_{i}$. Using Lemma 2, Proposition 4 for $\left(\tilde{X}_{1}, \tilde{X}_{2}\right)$, and then Proposition 6, yields

$$
\begin{aligned}
\operatorname{REv}\left(X_{1}, X_{2}\right) & \leq \lambda_{1} \frac{R_{1}}{\lambda_{1}}+\lambda_{2} \frac{R_{2}}{\lambda_{2}}+\frac{\lambda_{2}}{e} \frac{R_{1}}{\lambda_{1}}+\frac{\lambda_{1}}{e} \frac{R_{2}}{\lambda_{2}}+\frac{\lambda_{1}(e-1)}{e} \min \left\{\frac{R_{1}}{\lambda_{1}}, \frac{R_{2}}{\lambda_{2}}\right\} \\
& \leq R_{1}+R_{2}+\frac{1}{\lambda e} R_{1}+\frac{\lambda}{e} R_{2}+\frac{\lambda(e-1)}{e} R_{2} \\
& =R_{1}+R_{2}+\frac{1}{\lambda e} R_{1}+\lambda R_{2},
\end{aligned}
$$

where in the second line, we put $\lambda:=\lambda_{1} / \lambda_{2} \in(0,1]$ and used $\min \left\{R_{1} / \lambda_{1}, R_{2} / \lambda_{2}\right\} \leq R_{2} / \lambda_{2}$. The final expression equals $(1+1 / \sqrt{e})\left(R_{1}+R_{2}\right)$ when $\lambda=1 / \sqrt{e}$, completing the proof. ${ }^{24}$

\section{REGULAR GOODS AND MONOTONIC MECHANISMS}

In this section, we prove the second part of our Main Result, namely, the better bound of $73 \%$ for regular goods (and also for monotonic mechanisms). We will use here only the symmetric diagonal decomposition (i.e., $\lambda_{1}=\lambda_{2}=1$ ).

Following Myerson [1981], we say that a one-dimensional random variable $X$ is weakly regular if its support is an interval $[\alpha, \beta]$ with $^{25} 0 \leq \alpha<\beta \leq \infty$, on which it has a density function $f(t)$ that is positive and continuous, and the resulting "virtual valuation function" $t-G(t) / f(t)$ is nondecreasing (Myerson's regularity condition requires the virtual valuation to be strictly increasing).

Lemma 8. Assume that $X_{1}$ and $X_{2}$ are weakly regular. Then $K_{i}(u)>0$ implies that $K_{i}(v) \geq 0$ for all $v>u$, for $i=1,2$.

Proof. Let $\left[\alpha_{i}, \beta_{i}\right]$ be the support of $X_{i}$. Assume by way of contradiction that, say, $K_{1}(u)>0$ and $K_{1}(v)<0$ for some $v>u$. First, $K_{1}(u)>0$ implies that $f_{2}(u)>0$ and $H_{1}(u)-r_{1}>0$ (otherwise $K_{1}(u) \leq-G_{1}(u) G_{2}(u) \leq 0$ ), and so $\alpha_{2} \leq u \leq \beta_{2}$ and $u>\alpha_{1}$ (because $H_{1}$ is nondecreasing and $\left.H_{1}\left(\alpha_{1}\right) \leq \alpha_{1}=\alpha_{1} \cdot G_{1}\left(\alpha_{1}\right) \leq r_{1}\right)$. Second, $K_{1}(v)<0$ implies that $G_{1}(v)>0$ and $G_{2}(v)>0$ (otherwise $K_{1}(v)=f_{2}(v)\left(H_{1}(v)-r_{1}\right) \geq f_{2}(v)\left(H_{1}(u)-r_{1}\right) \geq 0$, since $H_{1}$ is nondecreasing), and so $v<\beta_{1}$ and $v<\beta_{2}$ (because $G_{i}\left(\beta_{i}\right)=0$ ). Together with $u<v$ it follows that $u$ and $v$ both lie in the interval $^{26}$ where $f_{2}(t)>0, G_{1}(t)>0$, and $H_{1}(t)-r_{1}>0$. But in that interval the function $\kappa$, defined by

$$
\kappa(t):=\frac{K_{1}(t)}{f_{2}(t) G_{1}(t)}=\left(\frac{H_{1}(t)-r_{1}}{G_{1}(t)}-t\right)+\left(t-\frac{G_{2}(t)}{f_{2}(t)}\right),
$$

\footnotetext{
${ }^{23}$ The results of the previous sections will be applied to the rescaled $\tilde{X}_{i}=X_{i} / \lambda_{i}$, and so we will use $r_{i}$ for the revenue of $\tilde{X}_{i}$, and $R_{i}$ for the revenue of the original $X_{i}$.

${ }^{24}$ One may check that $1+1 / \sqrt{e}$ is the best bound that is independent of $R_{1}$ and $R_{2}$ (when $R_{1}=R_{2}$ the above expression is minimized only at $\lambda=1 / \sqrt{e}$ ).

${ }^{25}$ Notice that we allow $\beta=\infty$, in which case the interval is understood to be $[\alpha, \infty)$.

${ }^{26}$ I.e., $t_{1}<u<v<\beta_{1}$, where $t_{1}>\alpha_{1}$ is the point where $H_{1}\left(t_{1}\right)=r_{1}$, and $\alpha_{2} \leq u<v<\beta_{2}$.
} 
is increasing-the derivative of the first term is $f_{1}(t)\left(H_{1}(t)-r_{1}\right) / G_{1}^{2}(t)>0$, and the second term is nondecreasing by regularity. Therefore, we cannot have $\kappa(u)>0$ and $\kappa(v)<0$, which contradicts the assumption that $K_{1}(u)>0$ and $K_{1}(v)<0$.

This yields the second part of our Main Result:

THEOREM 9. Let $X=\left(X_{1}, X_{2}\right)$ be a two-good random valuation with independent and weakly regular goods. Then

$$
\frac{\operatorname{SREV}\left(X_{1}, X_{2}\right)}{\operatorname{REV}\left(X_{1}, X_{2}\right)} \geq \frac{e}{e+1} \approx 0.73 .
$$

Proof. Take $\lambda_{1}=\lambda_{2}=1$ and let $r_{i}=\operatorname{Rev}\left(X_{i}\right)$. Lemma 8 implies that if $K_{i}(t)$ is positive anywhere, then it is nonnegative from that point on, and so either (i) there is some finite $u \geq 0$ such that $K_{i}(t) \leq 0$ for $t<u$ and $K_{i}(t) \geq 0$ for $t \geq u$ or (ii) $K_{i}(t) \leq 0$ for all $t \geq 0$. Therefore, for any function $\varphi_{i}$ with values in $[0,1]$, we have

$$
\int_{0}^{\infty} \varphi_{i}(t) K_{i}(t) \mathrm{d} t \leq \int_{u}^{\infty} K_{i}(t) \mathrm{d} t=L_{i}(u) \leq \frac{1}{e} r_{j}
$$

in case (i) (by Equations (18) and (19)), and $\int_{0}^{\infty} \varphi_{i}(t) K_{i}(t) d t \leq 0$ in case (ii). Altogether $\int \varphi_{1} K_{1}+$ $\int \varphi_{2} K_{2} \leq(1 / e) r_{2}+(1 / e) r_{1}$, and so Proposition 4 gives $\operatorname{Rev}(X) \leq(1+1 / e)\left(r_{1}+r_{2}\right)$, proving the result.

Next, let $\operatorname{Mon} \operatorname{Rev}(X)$ denote the maximal revenue that can be obtained using monotonic mechanisms, i.e., mechanisms $\mu=(q, s)$ for which the function $s(x)$ is nondecreasing in $x$.

Proposition 10. Let $X=\left(X_{1}, X_{2}\right)$ be a two-good random valuation with independent and weakly regular goods. Then

$$
\frac{\operatorname{SREV}\left(X_{1}, X_{2}\right)}{\operatorname{MONREV}\left(X_{1}, X_{2}\right)} \geq \frac{e}{e+1} \approx 0.73 .
$$

Proof. Put $r_{i}:=\operatorname{Rev}\left(X_{i}\right)$, and let $V_{i}$ be the "equal-revenue" (ER) random valuation with the same revenue $r_{i}$ as $X_{i}$; i.e., its tail distribution function is $\hat{G}_{i}(t)=\min \left\{r_{i} / t, 1\right\} \geq G_{i}(t)$. Take $V_{1}$ and $V_{2}$ to be independent, and put $V=\left(V_{1}, V_{2}\right)$. Because $V_{i}$ first-order stochastically dominates $X_{i}$, for every monotonic mechanism $\mu=(q, s)$, we have $R(\mu ; X)=\mathbb{E}\left[s\left(X_{1}, X_{2}\right)\right] \leq \mathbb{E}\left[s\left(V_{1}, V_{2}\right)\right]=R(\mu ; V)$. Therefore,

$$
\operatorname{MonRev}(X) \leq \operatorname{MonRev}(V) \leq \operatorname{Rev}(V) .
$$

The ER-good $V_{i}$ is weakly regular (because on its support $\left[r_{i}, \infty\right)$ the virtual valuation function $t-\hat{G}_{i}(t) / \hat{f}_{i}(t)$ is identically 0$)$, and $\operatorname{so} \operatorname{SRev}(V) \geq e /(e+1) \operatorname{Rev}(V)$ by Theorem 9; together with $\operatorname{SRev}(X)=\operatorname{SRev}(V)$ (by construction) and $\operatorname{Mon} \operatorname{Rev}(X) \leq \operatorname{Rev}(V)$ (see above), the result follows.

\section{APPENDICES}

\section{A REVENUE CONTINUITY}

This appendix deals with the continuity of the revenue with respect to valuations, which is of independent interest. Take a sequence of $k$-good valuations $X^{n}$ that converges in distribution to the $k$-good random valuation $X$; does the sequence of revenues $\operatorname{Rev}\left(X^{n}\right)$ converge to ${ }^{27} \operatorname{Rev}(X)$ ?

\footnotetext{
${ }^{27}$ Only the distribution of a random valuation $X$ matters for the revenue achievable from $X$; it is thus natural to consider what happens when $X^{n}$ converges in distribution to $X$. Formally, convergence in distribution is equivalent to the cumulative distribution functions converging pointwise at all points of continuity of the limit cumulative distribution. Informally, being close in distribution means that the probabilities of nearby values are close (see, for instance, Equation (24) below). Billingsley's [1968] book is a good reference for the concepts used here.
} 
Even in the one-good case this need not be so: for each $n$ let $X^{n}$ be the one-good valuation that takes value 0 with probability $1-1 / n$ and value $n$ with probability $1 / n$. Then $X^{n}$ converges in distribution to the valuation $X$ that takes value 0 with probability 1 . $\operatorname{But} \operatorname{REv}\left(X^{n}\right)=1$ (with the posted price of $n$ ) while $\operatorname{Rev}(X)=0$.

We will show that if the valuations all lie in a bounded set-more generally, if the random valuations are uniformly integrable-then the limit of the revenues equals the revenue of the limit. We emphasize that all the results in this Appendix are for general $k$-good valuations for any $k \geq 1$, whether the goods are independent or not. ${ }^{28}$

Some notation. First, it will be convenient to work here with the $\ell_{1}$-norm on ${ }^{29} \mathbb{R}^{k}$, i.e., $\|x\|_{1}=$ $\sum_{i=1}^{k}\left|x_{i}\right|$. The $\ell_{1}$-norm of a valuation $x$ in $\mathbb{R}_{+}^{k}$ provides a simple bound on the seller's payoff in any mechanism $\mu=(q, s)$ that is IR: $s(x) \leq q(x) \cdot x \leq \sum_{i} x_{i}=\|x\|_{1}$; thus, if a random valuation $X$ satisfies $\|X\|_{1} \leq M$, then $\operatorname{Rev}(X) \leq M$. Second, the Prohorov distance between the distributions of $X$ and $Y$, which we denote by $\operatorname{Dist}(X, Y)$, is defined as the infimum of all $\rho>0$ such that

$$
\begin{aligned}
& \mathbb{P}[X \in A] \leq \mathbb{P}\left[Y \in B_{\rho}(A)\right]+\rho, \text { and } \\
& \mathbb{P}[Y \in A] \leq \mathbb{P}\left[X \in B_{\rho}(A)\right]+\rho
\end{aligned}
$$

for all measurable sets $A$, where $B_{\rho}(A):=\left\{y:\|y-x\|_{1}<\rho\right.$ for some $\left.x \in A\right\}$ is the $\rho$-neighborhood of $A$. Thus, $0 \leq \operatorname{Dist}(X, Y) \leq 1$, and $X^{n}$ converges in distribution to $X$, which we write as $X^{n} \stackrel{\mathcal{D}}{\rightarrow} X$, if and only if $\operatorname{Dist}\left(X, X^{n}\right) \rightarrow 0$ [again, see Billingsley 1968].

The basic result is that in the bounded case the distance between the revenues of two random valuations is uniformly bounded by a function of the Prohorov distance between their distributions.

Proposition 11. Let $X$ and $Y$ be $k$-good valuations with bounded values, say, $\|X\|_{1},\|Y\|_{1} \leq M$ for some $M \geq 1$. Then ${ }^{30}$

$$
|\operatorname{REV}(X)-\operatorname{REV}(Y)| \leq(2 M+1) \sqrt{\operatorname{Dist}(X, Y)} .
$$

Proof. If $\operatorname{Dist}(X, Y)=1$ there is nothing to prove, since both revenues are between 0 and $M$.

Thus, let $0<\rho<1$ be such that Equation (24) holds for every measurable set $A \subseteq \mathbb{R}_{+}^{k}$, and take $\alpha$ so $\rho \leq \alpha<1$ (the value of $\alpha$ will be determined later). Denote by $D_{M}:=\left\{x \in \mathbb{R}_{+}^{k}:\|x\|_{1} \leq M\right\}$ the domain of values of $X$ and $Y$.

Let $\mu=(q, s)$ be an IC, IR, and NPT mechanism, and let $b$ be its buyer payoff function. We define a new mechanism $\tilde{\mu}$ by lowering all payments by a factor of $1-\alpha$ (and letting the buyer reoptimize). Thus, let $\mathrm{cl} W \subset \mathbb{R}_{+}^{k+1}$ be the closure of the set $W:=\left\{(q(x),(1-\alpha) s(x)): x \in D_{M}\right\}$. For each $x \in D_{M}$ let $(\tilde{q}(x), \tilde{s}(x))$ be a maximizer for ${ }^{31} \tilde{b}(x):=\max _{(g, t) \in \mathrm{cl} W}(g \cdot x-t)$. Then the mechanism $\tilde{\mu}=(\tilde{q}, \tilde{s})$ is IC (by the maximizer definition), IR (because $\tilde{b}(x) \geq b(x)+\alpha s(x)$, which is nonegative, since $\mu$ is IR and NPT), and NPT (because $\mu$ is NPT) ${ }^{32}$

Let $x, y \in D_{M}$ be such that $\|x-y\|_{1} \leq \rho$. Then $(\tilde{q}(y), \tilde{s}(y)) \in \mathrm{cl} W$ can be approximated by elements of $W$ : for every $\varepsilon>0$ there is $z \in D_{M}$ such that, in particular, $|\tilde{s}(y)-(1-\alpha) s(z)| \leq \varepsilon$ and

\footnotetext{
${ }^{28}$ Monteiro [2015] establishes continuity of the optimal revenue in the one-good case with $n$ independent buyers, when the valuations are bounded and the limit distributions are continuous (his proof uses the characterization of the optimal mechanism).

${ }^{29}$ This affects only the various constants below.

${ }^{30}$ We have not attempted to optimize the bound here.

${ }^{31}$ This maximum is attained because $W$ is bounded, namely, $W \subseteq[0,1]^{k} \times[0, M]$, and so cl $W$ is a compact set.

${ }^{32}$ Hart and Reny [2015] use this device of applying a small uniform discount to the buyer's payments to show that, at an arbitrarily small cost, the seller can perturb any IC and IR mechanism so the buyer breaks any indifference in the seller's favor (the resulting mechanism is thus called seller-favorable).
} 
$|[\tilde{q}(y) \cdot y-\tilde{s}(y)]-[q(z) \cdot y-(1-\alpha) s(z)]| \leq \varepsilon$. We then have

$$
\begin{aligned}
q(z) \cdot y-(1-\varepsilon) s(z)+\varepsilon & \geq \tilde{q}(y) \cdot y-\tilde{s}(y) \\
& \geq q(x) \cdot y-(1-\alpha) s(x) \\
& =q(x) \cdot x-s(x)+q(x) \cdot(y-x)+\alpha s(x) \\
& \geq q(z) \cdot x-s(z)+q(x) \cdot(y-x)+\alpha s(x),
\end{aligned}
$$

where the second inequality follows, because $(q(x),(1-\alpha) s(x)) \in W$, and the last inequality follows because $(q, s)$ is IC. Rearranging gives

$$
\alpha(s(z)-s(x)) \geq(q(x)-q(z)) \cdot(y-x)-\varepsilon .
$$

Now $|(q(x)-q(z)) \cdot(y-x)| \leq \rho$ (because $q(x), q(z) \in[0,1]^{k}$ and $\left.\|y-x\|_{1} \leq \rho\right)$, and so

$$
(1-\alpha) s(z) \geq(1-\alpha) s(x)-\frac{1-\alpha}{\alpha}(\rho+\varepsilon) \text {. }
$$

Recalling that $\tilde{s}(y) \geq(1-\alpha) s(z)-\varepsilon$ yields

$$
\tilde{s}(y) \geq(1-\alpha) s(x)-\frac{1-\alpha}{\alpha}(\rho+\varepsilon)-\varepsilon ;
$$

as $\varepsilon>0$ was arbitrary, we have

$$
\tilde{s}(y) \geq(1-\alpha) s(x)-\frac{1-\alpha}{\alpha} \rho,
$$

and so, using $s(x) \leq\|x\|_{1} \leq M$,

$$
\tilde{s}(y) \geq s(x)-\beta,
$$

where $\beta:=\alpha M+(1-\alpha) \rho / \alpha$.

For each $t>0$ put $A(t):=\left\{x \in D_{M}: s(x) \geq t\right\}$ and $\tilde{A}(t):=\left\{x \in D_{M}: \tilde{s}(x) \geq t\right\}$. Inequality (25), which applies whenever $\|y-x\|_{1} \leq \rho$, implies that $\tilde{A}(t-\beta) \supseteq B_{\rho}(A(t))$, and so

$$
\begin{aligned}
\operatorname{Rev}(Y) & \geq \mathbb{E}[\tilde{s}(Y)]=\int_{0}^{\infty} \mathbb{P}[Y \in \tilde{A}(t)] \mathrm{d} t \geq \int_{\beta}^{M} \mathbb{P}[Y \in \tilde{A}(t-\beta)] \mathrm{d} t \\
& \geq \int_{\beta}^{M} \mathbb{P}\left[Y \in B_{\rho}(A(t))\right] \mathrm{d} t \geq \int_{\beta}^{M} \mathbb{P}[X \in A(t)] \mathrm{d} t-(M-\beta) \rho \\
& \geq \int_{0}^{M} \mathbb{P}[X \in A(t)] \mathrm{d} t-\beta-(M-\beta) \rho=\mathbb{E}[s(X)]-\beta^{\prime},
\end{aligned}
$$

where $\beta^{\prime}:=\beta+(M-\beta) \rho$ (for the fourth inequality we have used Equation (24) and $\beta \leq M$, which follows from $\rho \leq \alpha$ and $M \geq 1)$.

Taking $\alpha=\sqrt{\rho}$ gives $\beta \leq(M+1) \sqrt{\rho}$ and $\beta^{\prime} \leq \beta+M \rho \leq(2 M+1) \sqrt{\rho}$. The displayed inequality above holds for every $\mu$ and every $\rho>\operatorname{Dist}(X, Y)$, and so $\operatorname{Rev}(Y) \geq \operatorname{Rev}(X)-(2 M+$ 1) $\sqrt{\operatorname{Dist}(X, Y)}$. Interchanging $X$ and $Y$ completes the proof.

A sequence of random variables $\left(X^{n}\right)_{n \geq 1}$ is uniformly integrable if for every $\varepsilon>0$ there is a finite $M$ such that $\mathbb{E}\left[\left\|X^{n}\right\|_{1} \mathbf{1}_{\left\|X^{n}\right\|_{1}>M}\right] \leq \varepsilon$ for all $n$.

THeorem 12. Let $X^{n}$ be a sequence of $k$-good random valuations that converges in distribution to the $k$-good random valuation $X$. Then

$$
\liminf _{n \rightarrow \infty} \operatorname{Rev}\left(X^{n}\right) \geq \operatorname{Rev}(X) .
$$

Moreover, if the sequence $X^{n}$ is uniformly integrable, then

$$
\lim _{n \rightarrow \infty} \operatorname{Rev}\left(X^{n}\right)=\operatorname{REv}(X)<\infty .
$$


Proof. For every $M>0$ and every $k$-good valuation $X$, denote $X_{(M)}:=X 1_{\|X\|_{1} \leq M}$. Any IR, IC, and NPT mechanism $\mu=(q, s)$ satisfies $s \geq 0$ and $s(0)=0$, and so $\mathbb{E}\left[s\left(X_{(M)}\right)\right]=\mathbb{E}\left[s(X) 1_{\|X\|_{1} \leq M}\right]$ monotonically increases to $\mathbb{E}[s(X)]$ as $M$ increases to infinity. Therefore, $\operatorname{REv}\left(X_{(M)}\right)$ monotonically increases to $\operatorname{Rev}(X)$.

If $X^{n} \stackrel{\mathcal{D}}{\rightarrow} X$, then $X_{(M)}^{n} \stackrel{\mathcal{D}}{\rightarrow} X_{(M)}$ for almost every $M>0$-specifically, for those $M$ where ${ }^{33}$ $\mathbb{P}\left[\|X\|_{1}=M\right]=0$-and so $\lim _{n} \operatorname{Rev}\left(X_{(M)}^{n}\right)=\operatorname{Rev}\left(X_{(M)}\right)$ by Proposition 11. Now $\operatorname{Rev}\left(X^{n}\right) \geq$ $\operatorname{Rev}\left(X_{(M)}^{n}\right)$, and hence $\lim \inf _{n} \operatorname{Rev}\left(X^{n}\right) \geq \operatorname{Rev}\left(X_{(M)}\right)$ for almost every $M$. Letting $M \rightarrow \infty$ proves the first part of the theorem.

If in addition the sequence $X_{n}$ is uniformly integrable, then for every $\varepsilon>0$ there is $M>0$ with $\mathbb{P}\left[\|X\|_{1}=M\right]=0$ that is large enough so $\mathbb{E}\left[\left\|X^{n}\right\|_{1} \mathbf{1}_{\left\|X^{n}\right\|_{1}>M}\right] \leq \varepsilon$ for all $n$. Since, as seen above, $0 \leq s(x) \leq\|x\|_{1}$ for every IR and NPT mechanism $\mu=(q, s)$, it follows that $\mathbb{E}\left[s\left(X^{n}\right) 1_{\left\|X^{n}\right\|_{1}>M}\right] \leq$ $\varepsilon$ for all $n$, and thus $\operatorname{Rev}\left(X_{(M)}^{n}\right) \geq \operatorname{Rev}\left(X^{n}\right)-\varepsilon$ for all $n$ (this also shows that the revenues are all finite, as they are bounded by $M+\varepsilon$ ). Therefore, $\operatorname{Rev}(X) \geq \operatorname{Rev}\left(X_{(M)}\right)=\lim _{n} \operatorname{Rev}\left(X_{(M)}^{n}\right) \geq$ $\lim \sup _{n} \operatorname{Rev}\left(X^{n}\right)-\varepsilon$, which, together with the first part of the theorem, proves the second part.

\section{A.1 Continuous Valuations}

It is often convenient-as in the present article-to restrict attention to random valuations whose distributions admit a density function (i.e., their cumulative distribution functions are absolutely continuous; we refer to these for now as "continuous"). We now show that for the results in the present article one may restrict attention to continuous random valuations. Indeed, assume that we have already proved a result of the form $\operatorname{Rev}(X) \leq \theta \operatorname{SRev}(X)$ for all such continuous $X$, and let $X$ be a valuation that is not necessarily continuous (and so it may have atoms and even finite support). First, because, as we have seen in the proof of Theorem 12 above, $\operatorname{Rev}\left(X_{(M)}\right) \rightarrow_{M \rightarrow \infty} \operatorname{Rev}(X)$ and $\operatorname{SRev}\left(X_{(M)}\right) \rightarrow_{M \rightarrow \infty} \operatorname{SRev}(X)$, it suffices to prove the result for random valuations $X$ with bounded values, say $\|X\|_{1} \leq M$. Second, let $U$ be independent of $X$ and distributed uniformly on $[0,1]^{k}$, and for every $n$ define $X^{n}:=X+(1 / n) U$. Then, clearly, the valuations $X^{n}$ are continuous, $X^{n} \stackrel{\mathcal{D}}{\rightarrow} X$, and the sequence $X^{n}$ is bounded $\left(\left\|X^{n}\right\|_{1} \leq\|X\|_{1}+(1 / n) k \leq M+k\right)$; therefore, $\operatorname{Rev}\left(X^{n}\right) \rightarrow_{n \rightarrow \infty} \operatorname{Rev}(X)$ and $\operatorname{SRev}\left(X^{n}\right) \rightarrow_{n \rightarrow \infty} \operatorname{SRev}(X)$ (apply the second part of Theorem 12 to the sequences $X^{n} \stackrel{\mathcal{D}}{\rightarrow} X$ and $X_{i}^{n} \stackrel{\mathcal{D}}{\rightarrow} X_{i}$ for all goods $\left.i\right)$. Thus, $\operatorname{Rev}(X) \leq \theta \operatorname{SREv}(X)$ holds for every bounded $X$, and so for every $X$.

\section{B NONSYMMETRIC DIAGONALS}

In this Appendix, we illustrate how the use of nonsymmetric diagonals alone may strictly improve the $50 \%$ bound of Hart and Nisan [2017], and, in some cases, also the $62 \%$ bound of our Theorem $7 .{ }^{34}$ However, this improvement is not uniform, in the sense that it does not yield a better constant $\operatorname{than}^{35} 50 \%$.

\footnotetext{
${ }^{33}$ These are the points of continuity of the cumulative distribution function of $\|X\|_{1}$; see Corollary 1 to Theorem 5.1 in Billingsley [1968].

${ }^{34}$ To get more than $50 \%$, the single-good revenues need just to be different, and to get more than $62 \%$ they need to be significantly so (with the revenue of one good about nine times higher than the revenue of the other).

${ }^{35}$ Yet another non-uniform bound may be obtained by optimizing $\lambda$ in the first line of Equation (22):
}

$$
\operatorname{Rev}\left(X_{1}, X_{2}\right) \leq R_{1}+R_{2}+\min \left\{\frac{2}{\sqrt{e}} \sqrt{R_{1} R_{2}}, \frac{2}{e} \sqrt{R_{1} R_{2}}+\left(1-\frac{1}{e}\right) \min \left\{R_{1}, R_{2}\right\}\right\},
$$

where $R_{i}=\operatorname{Rev}\left(X_{i}\right)$ for $i=1,2$. 
Proposition 13. Let $X=\left(X_{1}, X_{2}\right)$ be a two-good random valuation with independent goods. Then

$$
\operatorname{REv}\left(X_{1}, X_{2}\right) \leq\left(\sqrt{\operatorname{REv}\left(X_{1}\right)}+\sqrt{\operatorname{REv}\left(X_{2}\right)}\right)^{2} .
$$

Remark. When $\operatorname{Rev}\left(X_{1}\right) \neq \operatorname{Rev}\left(X_{2}\right)$ the right-hand side is strictly less than $2\left(\operatorname{Rev}\left(X_{1}\right)+\operatorname{Rev}\left(X_{2}\right)\right)$, the bound of Theorem A of Hart and Nisan [2017] (when $\operatorname{REv}\left(X_{1}\right)=\operatorname{Rev}\left(X_{2}\right)$ the two bounds are the same).

Proof. We follow the proof of Theorem A in Hart and Nisan [2017], but we now split the computation along the diagonal $Y=\lambda Z$ for some $\lambda>0$ (instead of splitting along $Y=Z$ ). The arguments in the proof there carry over, and, for each fixed value $z$ of $Z$, we now have

$$
\begin{aligned}
\mathbb{E}\left[s(Y, Z) 1_{Y \geq \lambda Z} \mid Z=z\right] & \leq \operatorname{Rev}(Y)+z \mathbb{P}[Y \geq \lambda z] \\
& =\operatorname{Rev}(Y)+\frac{1}{\lambda}(\lambda z) \mathbb{P}[Y \geq \lambda z] \\
& \leq \operatorname{Rev}(Y)+\frac{1}{\lambda} \operatorname{Rev}(Y)=\left(1+\frac{1}{\lambda}\right) \operatorname{Rev}(Y) .
\end{aligned}
$$

Similarly, for each fixed value $y$ of $Y$,

$$
\begin{aligned}
\mathbb{E}\left[s(Y, Z) \mathbf{1}_{Z \geq(1 / \lambda) Y} \mid Y=y\right] & \leq \operatorname{Rev}(Z)+y \mathbb{P}\left[Z \geq \frac{y}{\lambda}\right] \\
& =\operatorname{Rev}(Z)+\lambda \frac{y}{\lambda} \mathbb{P}\left[Z \geq \frac{y}{\lambda}\right] \\
& \leq \operatorname{Rev}(Z)+\lambda \operatorname{Rev}(Z)=(1+\lambda) \operatorname{Rev}(Z) .
\end{aligned}
$$

Taking expectation over the values of $Z$ and $Y$, adding the two inequalities, and then minimizing the resulting expression over $\lambda$ (by taking $\lambda=\sqrt{\operatorname{REv}(Y) / \operatorname{REv}(Z)}$ ) yields the result.

Remark. A better bound than the one of Proposition 13, albeit also non-uniform, has been obtained by Kupfer [2017].

\section{REFERENCES}

M. Babaioff, N. Immorlica, B. Lucier, and S. M. Weinberg. 2014. A simple and approximately optimal mechanism for an additive buyer. In Proceedings of the 55th Symposium on Foundations of Computer Science (FOCS'14). 21-30.

M. Babaioff, N. Nisan, and A. Rubinstein. 2018. Optimal deterministic mechanisms for an additive buyer. In Proceedings of the 19th ACM Conference on Economics and Computation (EC'18). 429.

P. Billingsley. 1968. Convergence of Probability Measures. Wiley.

P. Briest, S. Chawla, R. Kleinberg, and M. Weinberg. 2015. Pricing randomized allocations. f. Econ. Theor. 156 (2015), $144-174$.

S. Chawla, J. D. Hartline, and R. D. Kleinberg. 2007. Algorithmic pricing via virtual valuations. In Proceedings of the 8th ACM Conference on Electronic Commerce (EC'07). 243-251.

S. Chawla, D. L. Malec, and B. Sivan. 2010. The power of randomness in Bayesian optimal mechanism design. In Proceedings of the 11th ACM Conference on Electronic Commerce (EC'10). 149-158.

C. Daskalakis, A. Deckelbaum, and C. Tzamos. 2017. Strong duality for a multiple-good monopolist. Econometrica 85 (2017), 735-767.

S. Hart and N. Nisan. 2017. Approximate revenue maximization with multiple items. F. Econ. Theor. 172 (2017), 313-347.

S. Hart and N. Nisan. 2019. Selling multiple correlated goods: Revenue maximization and menu-size complexity. F. Econ. Theor. 183(2019), 991-1029.

S. Hart and P. J. Reny. 2015. Maximal revenue with multiple goods: Nonmonotonicity and other observations. Theoret. Econ. 10 (2015), 893-922.

R. Kupfer. 2017. A note on approximate revenue maximization with two items. arXiv 1712.03518 .

P. K. Monteiro. 2015. A note on the continuity of the optimal auction. Econ. Lett. 137 (2015), 127-130.

R. B. Myerson. 1981. Optimal auction design. Math. Op. Res. 6 (1981), 58-73.

R. T. Rockafellar. 1970. Convex Analysis. Princeton University Press.

J. Thanassoulis. 2004. Haggling over substitutes. f. Econ. Theor. 117 (2004), 217-245.

Received April 2018; revised October 2018; accepted June 2019

ACM Transactions on Economics and Computation, Vol. 7, No. 4, Article 18. Publication date: December 2019. 